TRANSACTIONS OF THE

AMERICAN MATHEMATICAL SOCIETY

Volume 359, Number 4, April 2007, Pages 1485-1498

S 0002-9947(06)04205-X

Article electronically published on November 3, 2006

\title{
A GENERALIZATION OF HALF-PLANE MAPPINGS TO THE BALL IN $\mathbb{C}^{n}$
}

\author{
JERRY R. MUIR, JR. AND TED J. SUFFRIDGE
}

\begin{abstract}
Let $F$ be a normalized $(F(0)=0, D F(0)=I)$ biholomorphic mapping of the unit ball $B \subseteq \mathbb{C}^{n}$ onto a convex domain $\Omega \subseteq \mathbb{C}^{n}$ that is the union of lines parallel to some unit vector $u \in \mathbb{C}^{n}$. We consider the situation in which there is one infinite singularity of $F$ on $\partial B$. In one case with a simple change-of-variables, we classify all convex mappings of $B$ that are half-plane mappings in the first coordinate. In the more complicated case, when $u$ is not in the span of the infinite singularity, we derive a form of the mappings in dimension $n=2$.
\end{abstract}

\section{INTRODUCTION}

In this article, the authors continue a classification started in [3] of biholomorphic mappings $F$ of the unit ball $B=B_{n}=\left\{z \in \mathbb{C}^{n}:\|z\|<1\right\}$ of $\mathbb{C}^{n}$ onto unbounded convex domains in $\mathbb{C}^{n}$ that can be written as the union of parallel lines. We assume, as is common, that $F$ is normalized to satisfy $F(0)=0$ and $D F(0)=I$, where $I$ is the identity operator.

We pause to standardize some notation. We write $\|\cdot\|$ for the Euclidean norm in $\mathbb{C}^{n}$ and $\langle\cdot, \cdot\rangle$ for the standard Hermitian inner product. The standard basis vectors of $\mathbb{C}^{n}$ are written $e_{k}, k=1, \ldots, n$. Given $z \in \mathbb{C}^{n}$, with $n \geq 2$, it will be convenient to write $z=\left(z_{1}, \hat{z}\right)$, where $\hat{z} \in \mathbb{C}^{n-1}$ contains the last $n-1$ components of $z$. Let $\mathbb{N}_{0}$ stand for the set of nonnegative integers. Then $\mathbb{N}_{0}^{n}$ is the set of multi-indices, and in common shorthand for $z \in \mathbb{C}^{n}$ and $\alpha \in \mathbb{N}_{0}^{n}$, we write

$$
z^{\alpha}=\prod_{k=1}^{n} z_{k}^{\alpha_{k}}, \quad|\alpha|=\sum_{k=1}^{n} \alpha_{k} .
$$

The group of biholomorphic automorphisms of $B$ is denoted Aut $B$. Furthermore, given a locally biholomorphic function $G: B \rightarrow \mathbb{C}^{n}$ and $\varphi \in$ Aut $B$, the Koebe transform of $G$ with respect to $\varphi$ is denoted $\Lambda_{\varphi}(G)$. More explicitly,

$$
\Lambda_{\varphi}(G)(z)=D \varphi(0)^{-1} D G(\varphi(0))^{-1}[G(\varphi(z))-G(\varphi(0))], \quad z \in B .
$$

If $\mathcal{K}$ is the family of all normalized biholomorphic convex mappings of $B$ into $\mathbb{C}^{n}$, then $\mathcal{K}$ is known 4 to be compact (in the topology of uniform convergence on compact subsets of $B$ ) and linearly invariant. (The latter meaning $\Lambda_{\varphi}(G) \in \mathcal{K}$ for

Received by the editors January 4, 2005.

2000 Mathematics Subject Classification. Primary 32H02; Secondary 30C55.

Key words and phrases. Biholomorphic, convex mapping, holomorphic automorphism.

(C)2006 American Mathematical Society Reverts to public domain 28 years from publication 
all $G \in \mathcal{K}$ and $\varphi \in$ Aut B.) Furthermore, each $G \in \mathcal{K}$ satisfies the useful growth condition

$$
\|G(z)\| \leq \frac{\|z\|}{1-\|z\|}, \quad z \in B .
$$

It was noted in 3 that if $\Omega=F(B)$ is unbounded, then the set $A=\{u \in \partial B$ : $r u \in \Omega, r \geq 0\}$ is nonempty. We are particularly interested in the case where $u,-u \in A$ for some $u \in \partial B$. (Incidentally, $A=\{u,-u\}$ is the only case that is possible if $A$ is disconnected.) Convexity implies that $\Omega$ is the union of lines parallel to the vector $u$. In [3], it is revealed that the family $\left\{\psi_{t}: t \in \mathbb{R}\right\} \subseteq$ Aut $B$ given by

$$
\psi_{t}(z)=F^{-1}(F(z)+t u), \quad z \in B, t \in \mathbb{R},
$$

is a one-parameter subgroup of Aut $B$. (The mapping $t \mapsto \psi_{t}$ is a continuous homomorphism of $\mathbb{R}$ into Aut $B$ with the topology of uniform convergence on compact subsets of $B$.) There exist $a, b \in \partial B$ for which $\lim _{t \rightarrow \infty} \psi_{t} \equiv a$ and $\lim _{t \rightarrow-\infty} \psi_{t} \equiv b$ uniformly on compact subsets of $B$. It is evident that $a$ and $b$ are infinite singularities of $F$. If $a \neq b$, it was shown that, up to a Koebe transform, $F$ has the form

$$
F(z)=\left(\frac{1}{2} \log \frac{1+z_{1}}{1-z_{1}}\right) e_{1}+K\left(\frac{\hat{z}}{\sqrt{1-z_{1}^{2}}}\right),
$$

where $K: B_{n-1} \rightarrow \mathbb{C}^{n}$ is holomorphic. This is clearly a generalization of a strip mapping of the unit disk.

We will now address what occurs when $a=b$, so that $F$ has only one singularity on $\partial B$. Assume that the singularity is $e_{1}$. If $u$ lies in the span of $e_{1}$, we will show that

$$
F(z)=\frac{z}{1-z_{1}}+K\left(\frac{\hat{z}}{1-z_{1}}\right)
$$

where $K: \mathbb{C}^{n-1} \rightarrow \mathbb{C}^{n}$ is a homogeneous polynomial of degree 2 . If $u$ lies outside of the span of $e_{1}$, then a more complicated situation results. In dimension $n=2$, we will prove that

$$
F(z)=\frac{z}{1-z_{1}}-\frac{1}{2}\left(\frac{z_{2}}{1-z_{1}}\right)^{2} e_{1}+K\left(\frac{z_{1}}{1-z_{1}}-\frac{1}{2}\left(\frac{z_{2}}{1-z_{1}}\right)^{2}-\frac{u_{1}}{u_{2}} \frac{z_{2}}{1-z_{1}}\right),
$$

where $K$ is holomorphic and $\mathbb{C}^{2}$-valued in a neighborhood of 0 in $\mathbb{C}$ with terms only of degree $\geq 2$.

These clearly generalize the half-plane mappings of the unit disk, and so it comes as no surprise that the familiar mappings

$$
z \mapsto \frac{z}{1-\langle z, v\rangle}, \quad z \in B
$$

(where $v \in \partial B$ ), are part of the classification. As a surprising corollary to our analysis, we find that these mappings are not extreme points of $\mathcal{K}$ when $n \geq 2$. (They are exactly the extreme points when $n=1$.)

The classification provided in this article addresses a portion of part (b) of the following conjecture, proposed by the authors in [3], and of which that paper addresses part (c). 
Conjecture 1.1. If $F: B \rightarrow \mathbb{C}^{n}$ is a normalized univalent holomorphic mapping of the ball onto a convex domain, then

(a) $F(B)$ is bounded and $F$ extends continuously to $\partial B$, or

(b) $F$ extends continuously to $\partial B$ except for one point that is an infinite discontinuity, or

(c) $F$ is given by (1.3), and $K$ extends continuously to $\partial B_{n-1}$.

\section{A Remark on the extreme points of $\mathcal{K}$}

A family of examples of unbounded convex mappings is furnished by the following lemma and leads to an interesting result.

Lemma 2.1. If $Q: \mathbb{C}^{n-1} \rightarrow \mathbb{C}$ is a homogeneous polynomial of degree 2 such that $\|Q\|=\sup _{\|w\| \leq 1}|Q(w)| \leq 1 / 2$, then the function $H: B \rightarrow \mathbb{C}^{n}$ given by

$$
H(z)=\frac{z}{1-z_{1}}+\left(\frac{Q(\hat{z})}{\left(1-z_{1}\right)^{2}}\right) e_{1}
$$

lies in $\mathcal{K}$.

Proof. It follows from Lemma 2.1 of [4] that $G: B \rightarrow \mathbb{C}^{n}$ defined by

$$
G(z)=z+Q(\hat{z}) e_{1}
$$

lies in $\mathcal{K}$. For $r \in(0,1)$, let $\varphi_{r} \in$ Aut $B$ be given by

$$
\varphi_{r}(z)=\frac{\left(z_{1}-r, \sqrt{1-r^{2}} \hat{z}\right)}{1-r z_{1}}, \quad z \in B
$$

Since $\mathcal{K}$ is a linearly invariant family, $\Lambda_{\varphi_{r}}(G) \in \mathcal{K}$ for each $r$. Now

$$
H=\lim _{r \rightarrow 1^{-}} \Lambda_{\varphi_{r}}(G),
$$

and therefore $H \in \mathcal{K}$ by compactness.

Corollary 2.2. The mappings (1.4) are not extreme points of $\mathcal{K}$ when $n \geq 2$.

Proof. Let $Q: \mathbb{C}^{n-1} \rightarrow \mathbb{C}$ be a homogeneous polynomial of degree 2 such that $\|Q\|=1 / 2$. The mapping $z \mapsto z /\left(1-z_{1}\right)$ can be written as the mean of the two mappings of the type (2.1) formed using $Q$ and $-Q$. Any other mapping of the form (1.4) is obtained by composition on the left and right by $U$ and $U^{*}$, respectively, for some unitary operator $U$ on $\mathbb{C}^{n}$.

Corollary 2.2 is somewhat unexpected, and not just because the mappings (1.4) are precisely the extreme points of $\mathcal{K}$ when $n=1$. If $G \in \mathcal{K}$ is written $G=$ $I+\sum_{k=2}^{\infty} P_{k}$, where each $P_{k}$ is a homogeneous polynomial of degree $k$, then $\left\|P_{k}\right\| \leq 1$ for all $k=2,3, \ldots$ (see [5]). Equality is achieved in these inequalities for every $k$ for any mapping of the form (1.4).

Example 2.3. The function

$$
H(z)=\frac{z}{1-z_{1}}-\frac{1}{2}\left(\frac{z_{2}}{1-z_{1}}\right)^{2} e_{1}, \quad z \in B,
$$

is of the form (2.1), and hence lies in $\mathcal{K}$. One can easily verify that $H(B)$ is the union of parallel lines in the direction $i e_{1}$ as well as the union of parallel lines in the direction $e_{2}$. It follows that $H(B)$ contains the real 2-dimensional space spanned by these vectors. This illustrates that there is not necessarily uniqueness in the choice of the direction vector. 


\section{The AUtomorphisms}

As described in the introduction, we consider an appropriate function $F$ such that the one-parameter group of automorphisms described in (1.2) tend to the same point on $\partial B$ as $t \rightarrow \pm \infty$. Without loss of generality, we may assume that $e_{1}$ is the fixed boundary point. (Indeed, replace $F$ by $U^{*} \circ F \circ U$ for an appropriately chosen unitary operator $U$ on $\mathbb{C}^{n}$.)

Recall [6] that the transformation

$$
T(z)=\frac{e_{1}+z}{1-z_{1}}, \quad z \in \mathbb{C}^{n}, z_{1} \neq 1,
$$

maps $B$ biholomorphically onto the Siegel right half-space $R=\left\{z \in \mathbb{C}^{n}: \operatorname{Re} z_{1}>\right.$ $\left.\|\hat{z}\|^{2}\right\}$, while sending $e_{1}$ to " $\infty$ ". If $\varphi \in$ Aut $B$ is such that $e_{1}$ is its only fixed point in $\bar{B}$, then $T \circ \varphi \circ T^{-1} \in$ Aut $R$ has $\infty$ as its only fixed point in $\bar{R}$. (By $\bar{R}$, we mean the closure of $R$ in the one-point compactification of $\mathbb{C}^{n}$.) It follows from Proposition 2.2.10 of [1] that

$$
T \circ \varphi \circ T^{-1}(z)=\left(z_{1}+a_{1}+2\langle V \hat{z}, \hat{a}\rangle, V \hat{z}+\hat{a}\right), \quad z \in R,
$$

for some choice of $a \in \partial R$ and unitary operator $V$ on $\mathbb{C}^{n-1}$ such that if $(I-V) w=\hat{a}$ for some $w \in \mathbb{C}^{n-1}$, then $2\langle w, \hat{a}\rangle \neq \bar{a}_{1}$.

For all $t \in \mathbb{R}$, the automorphism $\psi_{t}$ must satisfy (3.2) for some appropriate $a$ and $V$, and therefore we solve to find

$$
\psi_{t}(z)=\frac{\left(2 z_{1}+2\langle V \hat{z}, \hat{a}\rangle+\left(1-z_{1}\right) a_{1}, 2 V \hat{z}+2\left(1-z_{1}\right) \hat{a}\right)}{2+2\langle V \hat{z}, \hat{a}\rangle+\left(1-z_{1}\right) a_{1}}, \quad z \in B, t \in \mathbb{R}
$$

where $a$ and $V$ are functions of $t$ satisfying $a(0)=0$ and $V(0)=I$. It is useful to observe that, in fact, $a$ and $V$ are holomorphic in the real variable $t$. (That is, they are locally represented by a convergent power series in $t$.)

Lemma 3.1. There is an Hermitian operator $A$ on $\mathbb{C}^{n-1}$ such that $V(t)=e^{-i t A}$ for all $t \in \mathbb{R}$.

Proof. Let $\left\{\psi_{t}^{k}\right\}_{k=1}^{\infty}$ be the iterates of $\psi_{t}$ for any $t \in \mathbb{R}$. Inductively, this means that $\psi_{t}^{1}=\psi_{t}$ and $\psi_{t}^{k+1}=\psi_{t} \circ \psi_{t}^{k}$ for all positive integers $k$. Clearly, $\psi_{t}^{k}=\psi_{k t}$. Therefore $V(t)^{k}=V(k t)$ as a direct result of (3.3). Equivalently, $V(t)=V(t / k)^{k}$. Differentiation of this expression in $t$ yields

$$
V^{\prime}(t)=V\left(\frac{t}{k}\right)^{k-1} V^{\prime}\left(\frac{t}{k}\right)=V(t) V\left(\frac{t}{k}\right)^{-1} V^{\prime}\left(\frac{t}{k}\right) .
$$

Taking the limit of (3.4) as $k \rightarrow \infty$ gives the operator differential equation $V^{\prime}(t)=$ $V(t) V^{\prime}(0)$. Hence $V(t)=e^{t M}$ for some operator $M$ on $\mathbb{C}^{n-1}$. Clearly, $V(t)^{-1}=$ $V(-t)$ and $V(t)^{*}=e^{t M^{*}}$. Since $V(t)$ is unitary for all $t, M$ must be skew-Hermitian $\left(M^{*}=-M\right)$. The lemma follows by letting $A=i M$.

We will now perform some calculations to analyze the relationship between $a$ and $u$.

Differentiate the expression

$$
F(z)+t u=F\left(\psi_{t}(z)\right), \quad z \in B, t \in \mathbb{R},
$$


with respect to $t$ and choose $z=\psi_{t}^{-1}(0)$. (Then $z$ satisfies the equations $V \hat{z}=$ $-\left(1-z_{1}\right) \hat{a}$ and $z_{1}=\left(\|\hat{a}\|^{2}-a_{1} / 2\right)\left(1-z_{1}\right)$.) The result is

$$
u \equiv\left(i\langle A \hat{a}, \hat{a}\rangle-\left\langle\hat{a}, \hat{a}^{\prime}\right\rangle+a_{1}^{\prime} / 2, i A \hat{a}+\hat{a}^{\prime}\right)
$$

because $D F(0)=I$.

Setting the last $n-1$ components of (3.6) equal gives the first-order equation $\hat{a}^{\prime}+i A \hat{a} \equiv \hat{u}$. It follows that

$$
\hat{a}(t)=\sum_{k=1}^{\infty} \frac{(-i)^{k-1} t^{k} A^{k-1} \hat{u}}{k !} .
$$

Equating the first components of (3.6) reveals that $a_{1}^{\prime}(0)=2 u_{1}$. Using the facts that $\hat{a}^{\prime}(t)=e^{-i t A} \hat{u}, A \hat{a}(t)=i\left(e^{-i t A}-I\right) \hat{u}$, and $\hat{a}^{\prime \prime}(t)=-i A e^{-i t A} \hat{u}$, we differentiate to see that

$$
a_{1}^{\prime \prime}(t)=2\left\langle e^{-i t A} \hat{u}, \hat{u}\right\rangle .
$$

With initial values $a_{1}(0)=0$ and $a_{1}^{\prime}(0)=2 u_{1}$,

$$
\frac{a_{1}(t)}{2}=\left\langle\sum_{k=2}^{\infty} \frac{(-i)^{k-2} t^{k} A^{k-2} \hat{u}}{k !}, \hat{u}\right\rangle+t u_{1} .
$$

Recall that $\operatorname{Re} a_{1}(t)=\|\hat{a}(t)\|^{2}$ and therefore contains terms in $t$ only of degree $\geq 2$. This gives the following result.

Lemma 3.2. The component $u_{1}$ is purely imaginary. $\hat{u}$.

As is evident by Example 2.3, we cannot make any strong conclusions regarding

\section{MAPpings SUCH THAT $u$ LIES IN THE SPAN OF THE SINGUlaRity}

With the assumption that the boundary singularity is $e_{1}$, we assume that $\hat{u}=0$. Since $u_{1}$ is purely imaginary, $u=i e_{1}$. (The geometry of the situation renders the possibility $u=-i e_{1}$ redundant.) Furthermore, the work in the previous section leading to Lemma 3.2 implies $a_{1}=2 i t$ and $\hat{a}=0$. Accordingly, the form of the automorphisms in (3.3) is simplified to become

$$
\psi_{t}(z)=\frac{\left(z_{1}+i t\left(1-z_{1}\right), V \hat{z}\right)}{1+i t\left(1-z_{1}\right)}, \quad z \in B, t \in \mathbb{R} .
$$

We now prove the following.

Lemma 4.1. The function $F$ has the form

$$
F(z)=\left(\frac{z_{1}}{1-z_{1}}, \exp \left(\frac{z_{1}}{1-z_{1}} A\right) \frac{\hat{z}}{1-z_{1}}\right)+K\left(\exp \left(\frac{z_{1}}{1-z_{1}} A\right) \frac{\hat{z}}{1-z_{1}}\right),
$$

where $K$ is a holomorphic function of $n-1$ complex variables containing terms only of degree $\geq 2$ in its expansion about 0 .

Proof. Define the function $H: B \rightarrow \mathbb{C}^{n}$ by

$$
H(z)=\left(\frac{z_{1}}{1-z_{1}}, \exp \left(\frac{z_{1}}{1-z_{1}} A\right) \frac{\hat{z}}{1-z_{1}}\right) .
$$


Due to our above observations concerning $\psi_{t}$, we can calculate for all $z \in B$ and $t \in \mathbb{R}$

$$
\begin{aligned}
H\left(\psi_{t}(z)\right) & =\left(\frac{z_{1}+i t\left(1-z_{1}\right)}{1-z_{1}}, \exp \left(\frac{z_{1}+i t\left(1-z_{1}\right)}{1-z_{1}} A\right) \frac{V \hat{z}}{1-z_{1}}\right) \\
& =\left(\frac{z_{1}}{1-z_{1}}+i t, \exp \left(\frac{z_{1}}{1-z_{1}} A\right) \frac{\hat{z}}{1-z_{1}}\right) \\
& =H(z)+i t e_{1} .
\end{aligned}
$$

Let $G=F \circ H^{-1}$. (Clearly, $H$ is univalent.) For $w \in H(B)$, write $z=H^{-1}(w)$. Then

$$
G\left(w+i t e_{1}\right)=F\left(\psi_{t}(z)\right)=F(z)+i t e_{1}=G(w)+i t e_{1} .
$$

Define $K_{0}: H(B) \rightarrow \mathbb{C}^{n}$ to be given by $K_{0}(w)=G(w)-w$. Then in its expansion about $0, K_{0}$ contains terms only of degree $\geq 2$ in $w$. Furthermore,

$$
K_{0}\left(w+i t e_{1}\right)=K_{0}(w), \quad w \in H(B) .
$$

This indicates that $K_{0}$ is independent of the first coordinate of its argument. We may then define $K(\hat{w})=K_{0}(w)$ for $w \in H(B)$. It follows that $F(z)=w+K(\hat{w})$, as desired.

It is interesting to note that functions of the form given in Lemma 4.1 have the property that the image of the ball is the union of lines parallel to the vector $i e_{1}$. However, most will fail to be convex mappings. As we will show in the following lemmas, convexity implies that $A=0$ and $K$ is a homogeneous polynomial of degree 2 .

In the following, let $\Delta \subseteq \mathbb{C}$ be the open unit disk.

Lemma 4.2. If $\left(w_{1}, \zeta \hat{w}\right)+o(\zeta) \in F(B)$ for some $w \in \mathbb{C}^{n}$ and all $\zeta \in \bar{\Delta}$, then

$$
\left\|\frac{e^{-w_{1} A} \hat{w}}{1+w_{1}}\right\| \leq 1
$$

Proof. By comparing series terms, define $f: \bar{\Delta} \rightarrow B$ by

$$
f(\zeta)=F^{-1}\left(\left(w_{1}, \zeta \hat{w}\right)+o(\zeta)\right)
$$

to calculate

$$
\begin{aligned}
f(\zeta) & =F^{-1}\left(w_{1} e_{1}\right)+D F^{-1}\left(w_{1} e_{1}\right)(0, \hat{w}) \zeta+o(\zeta) \\
& =F^{-1}\left(\left(w_{1}, \zeta \hat{w}\right)+K(\zeta \hat{w})\right)+o(\zeta) \\
& =\left(\frac{w_{1}}{1+w_{1}}, e^{-w_{1} A} \frac{\zeta \hat{w}}{1+w_{1}}\right)+o(\zeta) .
\end{aligned}
$$

By Cauchy's estimates,

$$
\left\|\left(0, e^{-w_{1} A} \frac{\hat{w}}{1+w_{1}}\right)\right\|=\left\|f^{\prime}(0)\right\| \leq 1 .
$$

We now find that $V(t)=I$ for all $t \in \mathbb{R}$, which greatly simplifies the form of $F$.

Theorem 4.3. $A=0$. 
Proof. Let $\hat{v}$ be an eigenvector of $A$ with (real) eigenvalue $\lambda$, and assume $\|\hat{v}\|=1$. The result will follow once it is shown that $\lambda$ must be 0 .

Suppose that $\lambda<0$, and choose $c \in(0,1)$. We may choose $r \in(0,1)$ such that for any $x \in(r, 1)$,

$$
c \sqrt{\frac{1+x}{1-x}} \exp \left(\frac{\lambda x}{1-x}\right)<1
$$

For $x \in(r, 1)$ and $\zeta \in \bar{\Delta}$, define

$$
p_{1}=\left(x, c \zeta \sqrt{1-x^{2}} \hat{v}\right)
$$

Now $p_{1} \in B$ and

$$
F\left(p_{1}\right)=\left(\frac{x}{1-x}, c \zeta \sqrt{\frac{1+x}{1-x}} \exp \left(\frac{\lambda x}{1-x}\right) \hat{v}\right)+o(\zeta) .
$$

Define

$$
d=1-c \sqrt{\frac{1+x}{1-x}} \exp \frac{\lambda x}{1-x} .
$$

Then $d \in(0,1)$ and hence

$$
p_{2}=(0, d \zeta \hat{v})
$$

lies in $B$. Now $F\left(p_{2}\right)=(0, d \zeta \hat{v})+o(\zeta)$, and the convexity of $F(B)$ implies

$$
\frac{F\left(p_{1}\right)+F\left(p_{2}\right)}{2}=\left(\frac{x}{2(1-x)}, \frac{\zeta \hat{v}}{2}\right)+o(\zeta) \in F(B) .
$$

From Lemma 4.2, we see that

$$
\begin{aligned}
1 & \geq\left\|\zeta \exp \left(\frac{-x}{2(1-x)} A\right)\left(\frac{2(1-x)}{2-x}\right) \frac{\hat{v}}{2}\right\| \\
& =\left\|\zeta \exp \left(\frac{-\lambda x}{2(1-x)}\right) \frac{1-x}{2-x} \hat{v}\right\| .
\end{aligned}
$$

But (4.2) clearly tends to $\infty$ as $x \rightarrow 1^{-}$for nonzero $\zeta \in \bar{\Delta}$. This is a contradiction.

We now have that $\lambda \geq 0$. Suppose that $\lambda>0$. Let $p_{1}$ remain as defined in (4.1) with $c=\sqrt{1-x^{2}}$. The convexity of $F(B)$ implies that

$$
\frac{F\left(p_{1}\right)}{2}=\left(\frac{x}{2(1-x)}, \zeta(1+x) \exp \left(\frac{\lambda x}{1-x}\right) \frac{\hat{v}}{2}\right)+o(\zeta) \in F(B) .
$$

But Lemma 4.2 implies that

$$
\left\|\exp \left(\frac{\lambda x}{2(1-x)}\right) \frac{\zeta\left(1-x^{2}\right)}{2-x} \hat{v}\right\| \leq 1 .
$$

But as $x \rightarrow 1^{-}$, the left-hand side of (4.3) tends to $\infty$, a contradiction. Hence $\lambda=0$.

The next theorem completes our analysis of this case, and provides a nice final form for $F$.

Theorem 4.4. The function $K$ is a homogeneous polynomial of degree 2 .

Of course, this allows for the possibility that $K \equiv 0$. 
Proof. It remains to show that $K$ cannot contain terms of degree $\geq 3$. In a neighborhood of 0 , we may write

$$
K(\hat{w})=\sum_{\alpha \in \mathbb{N}_{0}^{n-1}} w^{\alpha} a_{\alpha}
$$

where the multi-indices have the form $\alpha=\left(\alpha_{2}, \ldots, \alpha_{n}\right)$, and $a_{\alpha} \in \mathbb{C}^{n}$ for each $\alpha \in \mathbb{N}_{0}^{n-1}$.

Let $z_{1}=r \in(0,1)$. Pick $\rho_{2}, \ldots, \rho_{n} \in(0,1)$ such that $\sum_{k=2}^{n} \rho_{k}^{2}<1 / 4$. Choose $z_{2}, \ldots, z_{n}$ so that $\left|z_{k}\right|=\rho_{k} \sqrt{1-r^{2}}$. This forces $\|z\|<(1+r) / 2$. Writing $w_{k}=$ $z_{k} /(1-r)$ for $k=2, \ldots, n$, we have

$$
F\left(z_{1}, z_{2} e^{i \theta_{2}}, \ldots, z_{n} e^{i \theta_{n}}\right)=\left(\frac{r}{1-r}, w_{2} e^{i \theta_{2}}, \ldots, w_{n} e^{i \theta_{n}}\right)+K\left(w_{2} e^{i \theta_{2}}, \ldots, w_{n} e^{i \theta_{n}}\right)
$$

for all $\theta_{2}, \ldots, \theta_{n} \in \mathbb{R}$.

Let $m$ be Lebesgue measure in $\mathbb{R}^{n-1}$ with the normalization $m\left([0,2 \pi]^{n-1}\right)=1$, and write $\theta=\left(\theta_{2}, \ldots, \theta_{n}\right) \in \mathbb{R}^{n-1}$. If $|\alpha| \geq 3$, then

$$
\int_{[0,2 \pi]^{n-1}} e^{-i \alpha \cdot \theta}\left(\frac{r}{1-r}, w_{2} e^{i \theta_{2}}, \ldots, w_{n} e^{i \theta_{n}}\right) d m(\theta)=0 .
$$

We can therefore calculate the Cauchy integrals

$$
\begin{aligned}
w^{\alpha} a_{\alpha} & =\int_{[0,2 \pi]^{n-1}} e^{-i \alpha \cdot \theta} K\left(w_{2} e^{i \theta_{2}}, \ldots, w_{n} e^{i \theta_{n}}\right) d m(\theta) \\
& =\int_{[0,2 \pi]^{n-1}} e^{-i \alpha \cdot \theta} F\left(z_{1}, z_{2} e^{i \theta_{2}}, \ldots, z_{n} e^{i \theta_{n}}\right) d m(\theta) .
\end{aligned}
$$

Using the growth bound (1.1), we have

$$
\left\|w^{\alpha} a_{\alpha}\right\| \leq \int_{[0,2 \pi]^{n-1}}\left\|F\left(z_{1}, z_{2} e^{i \theta_{2}}, \ldots, z_{n} e^{i \theta_{n}}\right)\right\| d m(\theta)<\frac{1+r}{1-r} .
$$

We now calculate

$$
\left|w^{\alpha}\right|=\prod_{k=2}^{n}\left|w_{k}\right|^{\alpha_{k}}=\left(\frac{1+r}{1-r}\right)^{|\alpha| / 2} \prod_{k=2}^{n} \rho_{k}^{\alpha_{k}} .
$$

Now (4.4) becomes

$$
\left\|a_{\alpha}\right\|\left(\frac{1+r}{1-r}\right)^{|\alpha| / 2} \prod_{k=2}^{n} \rho_{k}^{\alpha_{k}}<\frac{1+r}{1-r} .
$$

But $|\alpha| \geq 3$ implies

$$
\left\|a_{\alpha}\right\| \prod_{k=2}^{n} \rho_{k}^{\alpha_{k}}<\left(\frac{1-r}{1+r}\right)^{|\alpha| / 2-1} \rightarrow 0
$$

as $r \rightarrow 1^{-}$. Since the left-hand side of (4.5) is constant, $\left\|a_{\alpha}\right\|=0$. Therefore $K$ has no terms of degree $\geq 3$.

We now know the form of $F$. 
Theorem 4.5. If $\hat{u}=0$, then $F$ has the form

$$
F(z)=\frac{z}{1-z_{1}}+K\left(\frac{\hat{z}}{1-z_{1}}\right)
$$

where $K: \mathbb{C}^{n-1} \rightarrow \mathbb{C}^{n}$ is a homogeneous polynomial of degree 2 .

\section{MAPPINGS SUCH THAT $u$ FAILS TO LIE IN THE SPAN OF THE SINGULARITY}

In this more complicated situation, we restrict ourselves to dimension $n=2$. As in the last section, assume $\operatorname{Im} u_{1} \geq 0$. By composition of the form $U^{*} \circ F \circ U$, where $U z=\left(z_{1}, \gamma z_{2}\right),|\gamma|=1$, we may assume $u_{2}>0$. The operator $A$ is now a scalar $\lambda \in \mathbb{R}$. We shall prove the following.

Theorem 5.1. $\lambda=0$.

With this in place, we have the following form for $F$.

Theorem 5.2. If $n=2, \operatorname{Im} u_{1} \geq 0$, and $u_{2}>0$, then $F$ has the form

$$
F(z)=\frac{z}{1-z_{1}}-\frac{1}{2}\left(\frac{z_{2}}{1-z_{1}}\right)^{2} e_{1}+K\left(\frac{z_{1}}{1-z_{1}}-\frac{1}{2}\left(\frac{z_{2}}{1-z_{1}}\right)^{2}-\frac{u_{1}}{u_{2}} \frac{z_{2}}{1-z_{1}}\right),
$$

where $K$ is holomorphic in a neighborhood of 0 in $\mathbb{C}$ with terms only of degree $\geq 2$ in its expansion about 0.

Proof. Define $H: B \rightarrow \mathbb{C}^{2}$ by

$$
H(z)=\frac{z}{1-z_{1}}-\frac{1}{2}\left(\frac{z_{2}}{1-z_{1}}\right)^{2} e_{1}
$$

Since $\lambda=0$, we have $a_{1}(t)=2 t u_{1}+t^{2} u_{2}^{2}, a_{2}(t)=t u_{2}$, and $V(t)=1$ for all $t \in \mathbb{R}$. This allows the calculation

$$
\begin{aligned}
H\left(\psi_{t}(z)\right) & =\left(\frac{z_{1}}{1-z_{1}}+\frac{t u_{2} z_{2}}{1-z_{1}}+t u_{1}+\frac{t^{2} u_{2}^{2}}{2}-\frac{1}{2}\left(\frac{z_{2}}{1-z_{1}}+t u_{2}\right)^{2}, \frac{z_{2}}{1-z_{1}}+t u_{2}\right) \\
& =H(z)+t u
\end{aligned}
$$

for $z \in B$. Now $H$ is clearly univalent, and so define $G=F \circ H^{-1}$. Writing $w=H(z)$, we have

$$
G(w+t u)=F\left(\psi_{t}(z)\right)=F(z)+t u=G(w)+t u .
$$

Define $K_{0}: H(B) \rightarrow \mathbb{C}^{2}$ by $K_{0}(w)=G(w)-w$. Then in a neighborhood of $0, K_{0}$ has terms only of degree $\geq 2$. Implicitly define $K_{1}$ in a domain in $\mathbb{C}^{2}$ by the rule

$$
K_{1}\left(w_{1}-\frac{u_{1} w_{2}}{u_{2}}, w_{2}\right)=K_{0}(w)
$$

Then

$$
w+t u+K_{1}\left(w_{1}-\frac{u_{1} w_{2}}{u_{2}}, w_{2}\right)=G(w+t u)=w+t u+K_{1}\left(w_{1}-\frac{u_{1} w_{2}}{u_{2}}, w_{2}+t u_{2}\right),
$$

showing that $K_{1}$ is independent of its second component. Define a function $K$ in a domain in $\mathbb{C}$ to satisfy the relationship

$$
K\left(w_{1}-\frac{u_{1} w_{2}}{u_{2}}\right)=K_{1}\left(w_{1}-\frac{u_{1} w_{2}}{u_{2}}, w_{2}\right)
$$


so that

$$
G(w)=w+K\left(w_{1}-\frac{u_{1} w_{2}}{u_{2}}\right), \quad w \in H(B),
$$

as desired.

We devote the remainder of the section to proving Theorem 5.1. Assume that $\lambda \neq 0$. Then for all $t \in \mathbb{R}$,

$$
\begin{aligned}
& a_{1}(t)=2 t u_{1}-\frac{2 u_{2}^{2}}{\lambda^{2}}\left(e^{-i \lambda t}+i \lambda t-1\right), \\
& a_{2}(t)=-\frac{u_{2}}{i \lambda}\left(e^{-i \lambda t}-1\right), \\
& V(t)=e^{-i \lambda t}
\end{aligned}
$$

If $w \in \mathbb{C}$ satisfies $\left(1-e^{-i \lambda t}\right) w=a_{2}$, then $w=u_{2} /(i \lambda)$. The condition on $a$ and $V$ following (3.2) implies

$$
0 \neq 2 \bar{a}_{2} w-\bar{a}_{1}=2 t u_{1}-\frac{2 i t u_{2}^{2}}{\lambda}, \quad t \in \mathbb{R} .
$$

It follows that $u_{2}^{2}+i \lambda u_{1} \neq 0$. Therefore define the constant

$$
\rho=\frac{\lambda^{2}}{u_{2}^{2}+i \lambda u_{1}} .
$$

We shall now prove Theorem 5.1 through a sequence of lemmas.

Lemma 5.3. Define $g: B \rightarrow \mathbb{C}$ by

$$
g(z)=\frac{z_{1}}{1-z_{1}}+\frac{u_{2}}{i \lambda} \frac{z_{2}}{1-z_{1}} .
$$

Then for all $t \in \mathbb{R}$ and $z \in B$,

$$
g\left(\psi_{t}(z)\right)=g(z)-\frac{i \lambda t}{\rho} .
$$

Proof. The result follows from the simple calculation

$$
\begin{aligned}
g\left(\psi_{t}(z)\right) & =\frac{z_{1}}{1-z_{1}}+\frac{V \bar{a}_{2} z_{2}}{1-z_{1}}+\frac{a_{1}}{2}+\frac{u_{2}}{i \lambda}\left(\frac{V z_{2}}{1-z_{1}}+a_{2}\right) \\
& =\frac{z_{1}}{1-z_{1}}+\frac{u_{2}}{i \lambda} \frac{z_{2}}{1-z_{1}}+t u_{1}-\frac{i t u_{2}^{2}}{\lambda} \\
& =g(z)-\frac{i \lambda t}{\rho} .
\end{aligned}
$$

As is the case with functions of the form given in Lemma 4.1 the function $F$ given in the following lemma has the property that $F(B)$ is the union of lines parallel to $u$ even if $\lambda \neq 0$. However, for $F$ to be a convex mapping, Theorem 5.1 must hold.

Lemma 5.4. Define $H: B \rightarrow \mathbb{C}^{2}$ by

$$
H(z)=\left(1-\left[1-\frac{i \lambda}{u_{2}} \frac{z_{2}}{1-z_{1}}\right] e^{-\rho g(z)}\right)\left(\frac{u_{2}^{2}}{\lambda^{2}}, \frac{u_{2}}{i \lambda}\right)+\frac{i \rho g(z)}{\lambda} u .
$$


Then

$$
F(z)=H(z)+K\left(\frac{1}{\rho}\left(1-\left[1-\frac{i \lambda}{u_{2}} \frac{z_{2}}{1-z_{1}}\right] e^{-\rho g(z)}\right)\right),
$$

where $K$ is holomorphic in a domain in $\mathbb{C}$ and has terms only of degree $\geq 2$ in its expansion about 0.

Proof. We calculate for $t \in \mathbb{R}$ and $z \in B$,

$$
\begin{aligned}
H\left(\psi_{t}(z)\right)= & \left(1-\left[1-\frac{i \lambda}{u_{2}}\left(\frac{e^{-i \lambda t} z_{2}}{1-z_{1}}-\frac{u_{2}}{i \lambda}\left(e^{-i \lambda t}-1\right)\right)\right] e^{-\rho g(z)} e^{i \lambda t}\right)\left(\frac{u_{2}^{2}}{\lambda^{2}}, \frac{u_{2}}{i \lambda}\right) \\
& +\frac{i \rho g(z)}{\lambda} u+t u \\
= & H(z)+t u .
\end{aligned}
$$

Note that $H(0)=0$ and $D H(0)=I$. Furthermore, since $u_{2}^{2}+i \lambda u_{1} \neq 0$, the vectors $u$ and $\left(u_{2}^{2} / \lambda^{2}, u_{2} /(i \lambda)\right)$ are linearly independent. From that observation, it is easy to see that $H$ is univalent.

Now define $G=F \circ H^{-1}$. By the same technique as in the proof of Theorem 5.2 leading to (5.1), we may write

$$
G(w)=w+K\left(w_{1}-\frac{u_{1} w_{2}}{u_{2}}\right)
$$

where $w=H(z)$ and $K$ is analytic in a neighborhood of 0 in $\mathbb{C}$ and has terms only of degree $\geq 2$ in its expansion about 0 . The calculation

$$
w_{1}-\frac{u_{1} w_{2}}{u_{2}}=\frac{1}{\rho}\left(1-\left[1-\frac{i \lambda}{u_{2}} \frac{z_{2}}{1-z_{1}}\right] e^{-\rho g(z)}\right), \quad w=H(z), z \in B,
$$

gives the result.

Lemma 5.5. $\rho \geq 0$.

Proof. To the contrary, assume $\rho<0$. Then $\lambda>0$. We will begin by showing that $K$ given in Lemma 5.4 must be entire. It suffices to prove

$$
\left\{\left(1-\frac{i \lambda}{u_{2}} \frac{z_{2}}{1-z_{1}}\right) e^{-\rho g(z)}: z \in B\right\}=\mathbb{C} .
$$

To that end, write

$$
z=\left(\frac{t+i s}{1+t+i s},-\frac{i}{2} \frac{\sqrt{1+2 t}}{1+t+i s}\right) .
$$

Clearly $z \in B$ provided that $t>-1 / 2$ and $s \in \mathbb{R}$. With this substitution, the elements of the left-hand side of (5.3) have the form

$$
\left(1-\frac{\lambda \sqrt{1+2 t}}{2 u_{2}}\right) \exp \left(-\rho t+\frac{\rho u_{2} \sqrt{1+2 t}}{2 \lambda}\right) e^{-\rho i s} .
$$

The modulus of (5.4) can take on all nonnegative values, and the argument is unrestricted, proving that $K$ is entire.

Suppose, for the moment, that $K \equiv 0$. For $r \in[0,1)$, we have

$$
F(r, 0)=H(r, 0)=\left(1-\exp \frac{-\rho r}{1-r}\right)\left(\frac{u_{2}^{2}}{\lambda^{2}}, \frac{u_{2}}{i \lambda}\right)+\frac{i \rho r}{\lambda(1-r)} u .
$$


Applying the growth inequality (1.1) and multiplying by $\exp (\rho r /(1-r))$ gives

$$
\frac{r}{1-r} \exp \frac{\rho r}{1-r} \geq\left\|\left(\exp \frac{\rho r}{1-r}-1\right)\left(\frac{u_{2}^{2}}{\lambda^{2}}, \frac{u_{2}}{i \lambda}\right)+\frac{i \rho r}{\lambda(1-r)} \exp \left(\frac{\rho r}{1-r}\right) u\right\| \text {. }
$$

Taking the limit as $r \rightarrow 1^{-}$contradicts the assumption that $u_{2}>0$. It follows that $K \not \equiv 0$.

We now have that $K$ is nonzero and entire. Since $K(0)=0$ and $K^{\prime}(0)=0, K$ must contain terms only of degree $\geq 2$. Observe that

$$
\left\{\frac{1-e^{-\rho t} e^{-\rho i s}}{\rho}: t \geq 0, s \in \mathbb{R}\right\}=\mathbb{C} \backslash D\left(\frac{1}{\rho} ; \frac{1}{\rho}\right) .
$$

We can choose sequences $\left\{t_{k}\right\}$ and $\left\{s_{k}\right\}$, such that $t_{k} \geq 0$ and $s_{k} \in \mathbb{R}$ for all $k$, $t_{k} \rightarrow \infty$, and

$$
\left\|K\left(\frac{1-e^{-\rho t_{k}} e^{-i \rho s_{k}}}{\rho}\right)\right\| \geq c\left|\frac{1-e^{-\rho t_{k}} e^{-i \rho s_{k}}}{\rho}\right|^{2}
$$

for some constant $c>0$. Application of (1.1) gives

$$
\begin{aligned}
\sqrt{t_{k}^{2}+s_{k}^{2}} & \geq\left\|F\left(\frac{t_{k}+i s_{k}}{1+t_{k}+i s_{k}}, 0\right)\right\| \\
& \geq\left\|K\left(\frac{1-e^{-\rho t_{k}} e^{-i \rho s_{k}}}{\rho}\right)\right\|-\left\|H\left(\frac{t_{k}+i s_{k}}{1+t_{k}+i s_{k}}, 0\right)\right\| \\
& \geq c\left|\frac{1-e^{-\rho t_{k}} e^{-i \rho s_{k}}}{\rho}\right|^{2}-\left\|\left(1-e^{-\rho\left(t_{k}+i s_{k}\right)}\right)\left(\frac{u_{2}^{2}}{\lambda^{2}}, \frac{u_{2}}{i \lambda}\right)+\frac{i \rho\left(t_{k}+i s_{k}\right)}{\lambda} u\right\|
\end{aligned}
$$

for all $k$. Multiply through by $e^{2 \rho t_{k}}$ and take the limit as $k \rightarrow \infty$ to get $0 \geq 1 / \rho^{2}$, a contradiction.

Lemma 5.6. $\rho=0$.

Proof. Suppose $\rho>0$. Set

$$
E=\left\{z \in B: z_{2}=\frac{u_{2}\left(1-z_{1}\right)}{i \lambda}\right\}
$$

Now $z \in E$ must satisfy

$$
\left|1-z_{1}\right|^{2} \frac{u_{2}^{2}}{\lambda^{2}}<1-\left|z_{1}\right|^{2}
$$

It follows that

$$
\left|z_{1}\right|^{2}\left(1+\frac{u_{2}^{2}}{\lambda^{2}}\right)-\frac{2 u_{2}^{2}}{\lambda^{2}} \operatorname{Re} z_{1}<1-\frac{u_{2}^{2}}{\lambda^{2}}
$$

and so

$$
\left|z_{1}\right|^{2}-\frac{2 u_{2}^{2}}{\lambda^{2}+u_{2}^{2}} \operatorname{Re} z_{1}<\frac{\lambda^{2}-u_{2}}{\lambda^{2}+u_{2}} .
$$

Complete the square to find

$$
\left|z_{1}-\frac{u_{2}^{2}}{\lambda^{2}+u_{2}^{2}}\right|<\frac{\lambda^{2}}{\lambda^{2}+u_{2}^{2}}, \quad z \in E .
$$


This shows that for $z \in E, z_{1}$ can take on any value in the disk centered at $u_{2}^{2} /\left(\lambda^{2}+u_{2}^{2}\right)$ tangent to the unit circle at the point 1 . The image of this disk under $z_{1} \mapsto z_{1} /\left(1-z_{1}\right)$ is the half-plane $\left\{\zeta \in \mathbb{C}: \operatorname{Re} \zeta>\left(u_{2}^{2}-\lambda^{2}\right) /\left(2 \lambda^{2}\right)\right\}$. As a result,

$$
F(E)=\left\{\left(\frac{u_{2}^{2}}{\lambda^{2}}, \frac{u_{2}}{i \lambda}\right)+\frac{i \rho}{\lambda}\left(\zeta-\frac{u_{2}^{2}}{\lambda^{2}}\right) u+K\left(\frac{1}{\rho}\right): \operatorname{Re} \zeta>\frac{u_{2}^{2}-\lambda^{2}}{2 \lambda^{2}}\right\},
$$

using Lemma 5.4. To simplify, $F(E)$ (and hence $F(B)$ ) contains a ray of the form $\{c+i \rho s u / \lambda: s>0\}$ for some constant $c \in \mathbb{C}^{2}$. By convexity, $F(B)$ contains either $\{i s u: s>0\}$ or $\{i s u: s<0\}$ depending upon the sign of $\rho / \lambda$. However, since $\rho>0, \lambda s>0$ in either case. Since $F(B)$ contains $\{t u: t \in \mathbb{R}\}$, convexity implies that $\{\zeta u: \zeta \in P\} \subseteq F(B)$, where $P$ is a neighborhood of either the upper or lower closed half-plane in $\mathbb{C}$.

With $T$ given by (3.1), $z=e_{1}$, and $\varphi=\psi_{t}$, (3.2) becomes

$$
T \circ F^{-1}(t u)=\left(1+a_{1}(t), a_{2}(t)\right) .
$$

Since both sides of (5.5) are holomorphic in the real variable $t$,

$$
T \circ F^{-1}(\zeta u)=\left(1+a_{1}(\zeta), a_{2}(\zeta)\right)
$$

must hold for all $\zeta$ in some neighborhood of 0 in $\mathbb{C}$. (We extend $a_{1}$ and $a_{2}$ to a complex variable in the natural way.) Now the right-hand side of (5.6) is entire in $\zeta$, and the left-hand side is well defined for all $\zeta \in P$ by convexity. Hence (5.6) holds for all $\zeta \in P$ and takes on values in $R$. But if $\zeta=i s$ ( $s$ as determined by $P$ ), then the first coordinate of the right-hand side of (5.6) becomes

$$
1+a_{1}(i s)=1+2 i s u_{1}-\frac{2 u_{2}^{2}}{\lambda^{2}}\left(e^{\lambda s}-\lambda s-1\right) .
$$

Since $\lambda s>0$, (5.7) becomes negative as $|s| \rightarrow \infty$, a contradiction. Hence $\rho=0$.

Clearly, as $\rho=0, \lambda=0$, completing the proof of Theorem 5.1 .

\section{FINAL REMARKS}

It is interesting to note that the method of proof given in both Sections 4 and 5 is to define mappings of $B$ that are such that $F(B)$ is the union of parallel lines and then to impose restrictions on $F$ based on the hypothesis that $F(B)$ is convex. Hence we have constructed quite general mappings of $B$ such that the image of $B$ is the union of parallel lines but may not be convex.

Although we do not currently have a proof, we believe that Theorem 5.2 can be naturally extended to dimension $n>2$. In fact, it seems reasonable to conjecture that, under the assumption that $e_{1}$ is the infinite boundary singularity on $\partial B$, if $u$ is not in the span of $e_{1}, F(B)$ must still contain a line in the direction $i e_{1}$, and hence $F$ has the concise form given in Theorem 4.5.

\section{REFERENCES}

[1] Marco Abate, Iteration Theory of Holomorphic Maps on Taut Manifolds, Mediterranean Press, Rende, 1989. MR 1098711 (92i:32032)

[2] Steven G. Krantz, Function Theory of Several Complex Variables, 2nd ed., American Mathematical Society, Providence, 2001. MR1846625 (2002e:32001)

[3] Jerry R. Muir, Jr. and Ted J. Suffridge, Unbounded convex mappings of the ball in $\mathbb{C}^{n}$, Proc. Amer. Math. Soc., 129 (2001), no. 11, pp. 3389-3393. MR.1845017(2002f:32030)

[4] John A. Pfaltzgraff and Ted J. Suffridge, Linear invariance, order, and convex mappings in $\mathbb{C}^{n}$, Complex Variables Theory and Appl., 40 (1999), no. 1, pp. 35-50. MR1742869(2000i:32026) 
[5] John A. Pfaltzgraff and Ted J. Suffridge, Norm order and geometric properties of holomorphic mappings in $\mathbb{C}^{n}$, J. Anal. Math., 82 (2000), pp. 285 - 313. MR.1799667 (2001k:32028)

[6] Walter Rudin, Function Theory in the Unit Ball of $\mathbb{C}^{n}$, Springer-Verlag, New York, 1980. MR0601594(82i:32002)

Department of Mathematics, University of Scranton, Scranton, Pennsylvania 18510

E-mail address: muirj2@scranton.edu

Department of Mathematics, University of Kentucky, Lexington, Kentucky 40506

E-mail address: ted@ms.uky.edu 